\title{
Myasthenia gravis: a population based epidemiological study in Cambridgeshire, England
}

\author{
N P Robertson, J Deans, D A S Compston
}

\begin{abstract}
Objectives-To perform a comprehensive survey of myasthenia gravis in the county of Cambridgeshire, England, establishing contemporary epidemiological data.

Methods-Cases were ascertained from multiple sources. Prevalent patients were visited and assessed by means of a standardised questionnaire and examination complemented by review of medical case notes.

Results-One hundred cases were identified in a population of 684000 (prevalence 15 per 100000 population, 95\% confidence intervals (95\% CIs) 12-18). Thirty eight new diagnoses were made over a five year period providing an incidence of $1.1 / 100000$ population/year. The sex ratio was 2:1 F:M. After a mean follow up of 11.7 years, symptomatic disease was still restricted to ocular muscles in 25 patients. Thirty four of 100 patients underwent thymectomy a mean of 0.8 years after presentation, and a thymoma was present in 12 . Highest remission rates were seen in patients presenting with generalised disease who underwent thymectomy but did not have a thymoma (27\%). Cosegregation of an additional autoimmune disease occurred in 27 patients and in $24 / 49(49 \%)$ women with onset $<50$ years of age.

Conclusions-This, the second highest reported prevalence for myasthenia, is likely to be the result of optimum case ascertainment, increased disease duration, application of complex diagnostic tests, and the impact of an aging population leading to a relative increase in the prevalence of ocular myasthenia.
\end{abstract}

University of

Cambridge Neurology

Unit, Addenbrooke's

Hospital, Hills Rd, Cambridge CB2 2QQ, UK

N P Robertson

J Deans

D A S Compston

Correspondence to: Dr Neil Robertson, University of

Cambridge Neurology Unit,

Addenbrooke's Hospital,

Hills Rd, Cambridge

CB2 2QQ, UK. Telephone

00441223 216751; fax 0044

1223336941 .

Received 24 October 1997

and in final form

1 April 1998

Accepted 6 April 1998

Keywords: myasthenia gravis; prevalence; incidence

Although generally considered to be one of the rarer neurological diseases, myasthenia gravis is important because of advances made in understanding its immunological basis and the potential for treatment. Epidemiological studies which take into account advances in laboratory investigations allow the changing patterns of disease to be determined, provide information for health service planning, and allow the testing of aetiological hypotheses. These show a trend of increasing prevalence ${ }^{1}$ with relatively stable incidence which is interpreted as reflecting the impact of effective treatment and improved diagnostic methods. Wide fluc- tuations between geographical regions in morbidity statistics are seen in more recent examinations of disease frequency, and seem unlikely to be the result of these factors or study methodology alone.

Accurate epidemiological analysis of low frequency diseases requires a large population to identify significant numbers of affected people to disclose patterns of clinical phenotype. As a result, population based studies are scarce and most clinical reports on myasthenia gravis rely on consecutive case series culled from specialist neurology clinics which may not be entirely representative of the at risk population. We have applied an established procedure for epidemiological studies of neurological disease to survey a large population for myasthenia gravis and to examine patterns of clinical practice and disease phenotype.

\section{Patients and methods}

STUDY AREA

Cambridgeshire county lies within the East Anglian region of England. Neurological outpatient facilities are provided from district general hospitals in Peterborough and Huntingdon, which lie within the county boundaries; further outpatient facilities are available in Bury St Edmunds, Bedford, and Kings Lynn, which lie in adjoining counties. Each hospital is served by at least one consultant neurologist and their staff, and all inpatient investigation is performed at a single regional neurology unit in Cambridge, where records are held for all patients seen since 1965 . The county and adjoining area are served by 426 general practitioners operating from 154 surgeries. Population figures for the study area are published by the Cambridgeshire County Council Population Research Group, and report a 1995 mid-year estimate of $684000 .^{2}$
METHODS

A provisional register was created from eight sources. Departmental notes were examined to identify people seen since 1965 in whom a diagnosis of myasthenia had been made; this included records from neurophysiological examinations. Seven additional sources were used to identify patients with myasthenia gravis. All general practitioners within the Cambridgeshire, Huntingdon, and Peterborough County Districts and adjoining areas were asked to provide names, addresses, and dates of birth of affected patients registered with the practice and permission was sought to approach these patients. Reminders were sent 
Table 1 Recruitment sources for 100 prevalent patients

\begin{tabular}{|c|c|c|c|c|c|c|}
\hline \multirow[b]{2}{*}{ Recruitment source } & \multicolumn{2}{|c|}{$\begin{array}{l}\text { Preliminary } \\
\text { register } \\
(n=205)\end{array}$} & \multicolumn{2}{|c|}{$\begin{array}{l}\text { Prevalent } \\
\text { register } \\
(n=100)\end{array}$} & \multicolumn{2}{|c|}{$\begin{array}{l}\text { Sole source in } \\
\text { prevalent register } \\
(n=53)\end{array}$} \\
\hline & $n$ & $\%$ & $n$ & $\%$ & $n$ & $\%$ \\
\hline General practices & 95 & 46 & 78 & 78 & 42 & 42 \\
\hline Hospital database & 104 & 51 & 42 & 42 & 1 & 1 \\
\hline Departmental notes & 63 & 31 & 30 & 30 & 8 & 8 \\
\hline Myasthenia Gravis Association & 17 & 8 & 14 & 14 & 2 & 2 \\
\hline Immunology & 14 & 7 & 5 & 5 & 0 & 0 \\
\hline Thoracic surgery & 10 & 5 & 1 & 1 & 0 & 0 \\
\hline Ophthalmology & 5 & 2 & 3 & 3 & 0 & 0 \\
\hline Paediatric neurology & 1 & 0 & 0 & 0 & 0 & 0 \\
\hline Other specialties & 2 & 1 & 1 & 1 & 0 & 0 \\
\hline
\end{tabular}

at two 3-monthly intervals to non-responding practitioners. The local branch secretary of the Myasthenia Gravis Society was asked to provide a list of affected members once permission had been obtained from those concerned. The local thoracic surgery service provided names of patients who had undergone thymectomy for myasthenia gravis and similar requests were made to the paediatric neurologists and ophthalmologists. A list of requests for acetylcholine receptor antibodies since 1980 was obtained from a computerised record system held in the department of immunology and these patients' case notes were reviewed. Finally, computerised hospital databases provided a list of those patients admitted with a primary or secondary diagnosis of myasthenia gravis to Peterborough, Hinchingbrooke, Kings Lynn, or Addenbrooke's Hospitals after 1985. Patients were considered prevalent if they were alive and normally resident in the area on 1 July 1997, and incident when the diagnosis of myasthenia had been made while they were resident in the study area.

After establishing that the patient was aware of the diagnosis, and with consent from the general practitioner, attempts were made to contact all patients on the provisional register to confirm residence within the study area and to collect clinical data by examination and a standardised questionnaire complemented by review of hospital or primary case notes. The diagnosis of myasthenia was based on three or more of the following: (1) typical history; (2) clinical evidence of fatiguability with recovery on rest; (3) clinical response to anticholinesterase administration; (4) detection of acetylcholine receptor antibodies; (5) decrement on electrical activity on repetitive stimulation; (6) exclusion of alternative relevant diagnoses. Disease classification was according to modified Osserman group definitions. ${ }^{3}$ New diagnoses of myasthenia made by Cambridge based neurologists for the study area were recorded prospectively on a central register from 1992.

\section{Results}

CASE ASCERTAINMENT

The provisional register contained 205 patients from all sources. Many were identified from more than one source (table 1). One hundred and fifty one (98\%) of the 154 general practioner practices responded to the request for information. Ninety seven $(47 \%)$ patients on the provisional register had either died, moved away from the area, were duplicate referrals, or an alternative diagnosis had been established, and these were all excluded. After further clinical evaluation based on personal interview or review of existing medical records, the diagnosis could not be confirmed in a further eight $(4 \%)$ patients: alternate diagnoses comprised mitochondrial cytopathy $(n=3)$, eyelid apraxia $(n=1)$, idiopathic bulbar palsy $(n=1)$, and focal dystonia $(n=1)$; in a further two patients there was insufficient evidence to support the diagnosis of myasthenia even though no alternative seemed more likely.

The final register therefore comprised 100 patients with myasthenia gravis, alive and prevalent within the study area on 1 July 1997. The largest source for ascertainment was from general practitioner referrals (table 1), which also provide the largest number of patients identified from a single source $(42,42 \%$; see table 1$)$. Sixty one $(61 \%)$ of the 100 prevalent patients were examined and interviewed and the remainder assessed on the basis of recent and comprehensive departmental notes.

\section{PREVALENCE}

The prevalence of myasthenia gravis in Cambridgeshire on 1 July 1997 was $100 / 684000$ population (15/100 000 population; 95\% CI 12-18). Table 2 shows the age and sex specific prevalence of myasthenia gravis in Cambridgeshire, which varies from $0 / 100000$ in the $0-10$ year age group to $63 / 100000$ population (males 86, females 53) in the $80+$ age group. The sex ratio was $2: 1 \mathrm{~F}: \mathrm{M}$. The mean age of prevalent patients was 59.7 (males 68.2, females 55.5, range 16-89) years; mean age at onset was 46.0 (males 59.0 females 39.4) years, mean age at presentation was 47.5 (males 60.8, females 40.7) years, and mean age at diagnosis 47.8 (females 41.1, males 70.0) years. Fifty six $(56 \%)$ were aged 50 years or younger at diagnosis (males 7/33, 24\%; females 49/67, $73 \%$ ). A bimodal pattern of age at onset was

Table 2 Age and sex specific prevalence

\begin{tabular}{lccccccrrr}
\hline Age & Male & $n$ & Prevalence & Female & \multicolumn{1}{c}{$n$} & Prevalence & All & $n$ & Prevalence \\
\hline $0-10$ & 50500 & 0 & 0 & 47700 & 0 & 0 & 98200 & 0 & 0 \\
$11-20$ & 46700 & 0 & 0 & 43400 & 1 & 2 & 90100 & 1 & 1 \\
$21-30$ & 55400 & 0 & 0 & 51100 & 6 & 12 & 106500 & 6 & 6 \\
$31-40$ & 52500 & 2 & 4 & 50100 & 10 & 20 & 102600 & 12 & 12 \\
$41-50$ & 47400 & 3 & 6 & 47500 & 10 & 21 & 94900 & 13 & 14 \\
$51-60$ & 35200 & 5 & 14 & 34700 & 14 & 40 & 69900 & 19 & 27 \\
$61-70$ & 27800 & 4 & 14 & 29600 & 6 & 20 & 57400 & 10 & 17 \\
$71-80$ & 18300 & 13 & 71 & 24000 & 12 & 50 & 42300 & 25 & 59 \\
$\geqslant 81$ & 7000 & 6 & 86 & 15100 & 8 & 53 & 22100 & 14 & 63 \\
Total & 340800 & 33 & 10 & 343200 & 67 & 20 & 684000 & 100 & 15 \\
\hline
\end{tabular}




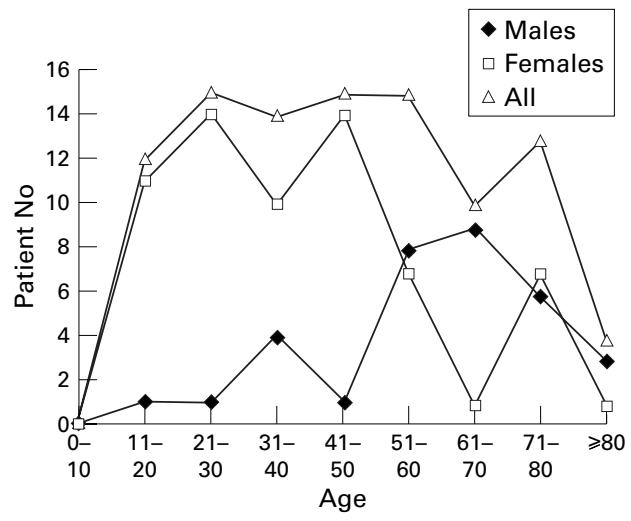

Age specific onset for 100 prevalent patients.

seen in females peaking at $21-50$ and $71-80$ years together with a single peak in males at 61-70 (figure).

INCIDENCE

In a five year period between 30 June 1992 and 1 July 1997 Cambridge based neurologists made 58 new diagnoses of myasthenia gravis, of which 38 lived within the study area; this provides a crude incidence of $11.1 /$ million population/year.

\section{CLINICAL CHARACTERISTICS}

At presentation, 52/100 patients had ocular and 48/100 more generalised disease. The commonest presenting symptoms and signs were ptosis $(64 \%)$ and diplopia (64\%). In six $(6 \%)$ patients, the delay from presentation to diagnosis was $>1$ year and initial erroneous diagnoses included motor neuron disease $(n=1)$, dysthyroid eye disease $(n=1)$, functional $(n=2)$, or vocal dystonia $(n=1)$. One patient was lost to follow up. Eight/100 (8\%) patients had precipitating factors for initial disease expression of which the commonest were drugs $(n=4)$, pregnancy $(n=2)$, or infection $(n=2)$. Eighty two/100 patients were known to have

Table 3 Diagnostic classification in 100 prevalent patients with myasthenia gravis

\begin{tabular}{|c|c|c|c|c|c|c|}
\hline \multirow[b]{2}{*}{ Osserman } & \multicolumn{2}{|c|}{ Male } & \multicolumn{2}{|c|}{ Female } & \multicolumn{2}{|l|}{ All } \\
\hline & $n$ & $\%$ & $n$ & $\%$ & $n$ & $\%$ \\
\hline I & 14 & 42 & 12 & 18 & 26 & 26 \\
\hline IIa & 5 & 15 & 12 & 18 & 17 & 17 \\
\hline IIb & 6 & 18 & 24 & 36 & 30 & 30 \\
\hline III & 5 & 15 & 6 & 9 & 11 & 11 \\
\hline IV & 3 & 9 & 13 & 19 & 16 & 16 \\
\hline Totals & 33 & & 67 & & 100 & \\
\hline
\end{tabular}

Table 4 Cosegregation of autoimmune disease in 100 prevalent patients with myasthenia gravis

\begin{tabular}{lllllll}
\hline & \multicolumn{2}{c}{ Males $(n(\%))$} & Females $(n(\%))$ & \multicolumn{2}{c}{ All $(n(\%))$} \\
\hline Thyrotoxicosis & 0 & 0 & 8 & 12 & 8 & 8 \\
Rheumatoid arthritis & 1 & 3 & 3 & 5 & 4 & 4 \\
Systemic lupus erythematosus & 0 & 0 & 4 & 6 & 4 & 4 \\
Hypothyroidism & 0 & 0 & 2 & 3 & 2 & 2 \\
Seronegative arthritis & 0 & 0 & 3 & 5 & 3 & 3 \\
Beçhet's & 0 & 0 & 1 & 2 & 1 & 1 \\
Vitiligo & 0 & 0 & 1 & 2 & 1 & 1 \\
Insulin dependent diabetes & 0 & 0 & 1 & 2 & 1 & 1 \\
Pemphigoid & 0 & 0 & 1 & 2 & 1 & 1 \\
Uveitis & 1 & 3 & 1 & 2 & 2 & 2 \\
Any autoimmune disease & 2 & 6 & 25 & 38 & 27 & 27 \\
\hline
\end{tabular}

undergone a tensilon test at presentation and in $76 / 82(93 \%)$ there was an unequivocal response. In $62 / 100$ patients the results of acetylcholine receptor antibody assays were documented and positive in $50 / 62(79 \%$; male $18 / 23$; 78 : female $32 / 39 ; 82 \%$ ). The mean disease duration was 13.7 years, and mean duration of follow up 11.9 years. Forty two/100 $(42 \%)$ patients were under current neurological review and four were under the care of other specialists (ophthalmology $(n=2)$ and general medicine $(n=2)$ ).

\section{COURSE}

Seventy five/100 patients had developed generalised disease at some stage and in 21/75 (28\%) this was restricted to oculobulbar or facial symptoms. Of the fifty two patients who presented with pure ocular disease, 27/52 (52\%) had gone on to develop generalised myasthenia. Patients had been admitted on average 1.9 times for investigation or control of disease. Fifty eight/100 had received immunosupressive therapy for a mean of 4.9 years. Fifty seven had received oral steroids, 17 azathiaprine, and only three plasmapheresis. Clinical classification according to the Osserman criteria identified 26/100 patients with grade I, 17 with grade IIa, 30 with grade IIb, II with grade III, and 16 with grade IV disease (table 3). The commonest side effects of treatment were anticholinergic effects of anticholinesterases severe enough to cause treatment modification $(n=12)$, cataracts $(n=3)$, recurrent infections $(n=2)$, azathiaprine related neutropoenia $(n=2)$, and osteoporosis $(n=4)$. Twenty seven/ $100(27 \%)$ patients had an additional history of autoimmune disease (males $2 / 33,6 \%$; females $25 / 67,38 \%$, table 4 ).

At the time of last review, $45 / 100$ patients were asymptomatic. Sixty four/100 were currently taking anticholinesterases and 41/100 were being treated with immunosupressive therapy. Thirty six/100 thought that their disease had represented a major handicap interfering with social or professional life.

Thirty five/ 100 patients (males $8 / 35,23 \%$; females $27,77 \%$ ) underwent a thymectomy after a mean delay of 0.8 years from presentation. Patients with thymoma related myasthenia tended to have more severe disease than non-thymomatous patients (Osserman grade III or IV $7 / 13 ; 53 \%$, table 5 ). Mean age at thymectomy was 27.8 years for those without and 39.6 years for those with a thymoma. Twelve patients had a thymoma and in three myasthenic symptoms postdated thymectomy by a mean of 2.2 years. One of $12(8 \%)$ patients who had undergone thymectomy for thymoma and $6 / 22(27 \%)$ without thymomas were currently in remission (stable and asymptomatic off treatment). This compares with $3 / 41$ $(7 \%)$ patients presenting with generalised disease who did not undergo thymectomy and $6 / 25(24 \%)$ presenting with ocular disease (table 5).

\section{Discussion}

It is generally held that the frequency of myasthenia is low irrespective of geographical, 


\begin{tabular}{|c|c|c|c|c|c|c|c|c|c|c|}
\hline & \multicolumn{2}{|c|}{ Thymoma } & \multicolumn{2}{|c|}{$\begin{array}{l}\text { Non-thymomatous } \\
\text { thymectomy }\end{array}$} & \multicolumn{2}{|c|}{$\begin{array}{l}\text { Generalised no } \\
\text { thymectomy }\end{array}$} & \multicolumn{2}{|c|}{$\begin{array}{l}\text { Ocular no } \\
\text { thymectomy }\end{array}$} & \multicolumn{2}{|c|}{ All patients } \\
\hline & $n$ & $\%$ & $n$ & $\%$ & $n$ & $\%$ & $n$ & $\%$ & $n$ & $\%$ \\
\hline No & 12 & 12 & 22 & 22 & 41 & 41 & 25 & 25 & 100 & 100 \\
\hline Male & 5 & 42 & 1 & 5 & 14 & 34 & 14 & 56 & 33 & 33 \\
\hline Female & 7 & 58 & 21 & 95 & 27 & 66 & 11 & 44 & 67 & 67 \\
\hline Mean age at presentation & 39.5 & - & 27.2 & - & 51.4 & - & 63.4 & - & 47.5 & - \\
\hline Osserman I & 1 & 8 & 0 & 0 & 0 & 0 & 25 & 100 & 26 & 26 \\
\hline Osserman IIa & 1 & 8 & 1 & 5 & 15 & 37 & 0 & 0 & 17 & 17 \\
\hline Osserman IIb & 3 & 25 & 10 & 45 & 17 & 41 & 0 & 0 & 30 & 30 \\
\hline Osserman III & 5 & 42 & 3 & 14 & 3 & 7 & 0 & 0 & 11 & 11 \\
\hline Osserman IV & 2 & 17 & 8 & 36 & 6 & 15 & 0 & 0 & 16 & 16 \\
\hline Remission (asymptomatic and stable \pm treatment) & 4 & 33 & 12 & 55 & 15 & 37 & 14 & 56 & 45 & 45 \\
\hline Remission (asymptomatic and stable off treatment) & 1 & 8 & 6 & 27 & 3 & 7 & 6 & 24 & 16 & 16 \\
\hline Current antiCE & 8 & 67 & 11 & 50 & 32 & 78 & 13 & 52 & 64 & 64 \\
\hline Current steroids & 8 & 67 & 7 & 32 & 19 & 46 & 6 & 24 & 40 & 40 \\
\hline Current azathiaprine & 4 & 33 & 3 & 14 & 3 & 7 & 3 & 12 & 13 & 13 \\
\hline Mean time on immunosupression (y) & 4.4 & - & 3.5 & - & 2.8 & - & 0.8 & - & 3.0 & - \\
\hline Mean No of hospital admissions & 4 & - & 3 & - & 1 & - & 0.8 & - & 1.9 & - \\
\hline
\end{tabular}

Table 6 Comparison of contemporary population based studies

\begin{tabular}{|c|c|c|c|c|c|c|}
\hline Area & Year & Population & $\begin{array}{l}\text { Patients } \\
\text { (n) }\end{array}$ & $\begin{array}{l}\text { Prevalence } \\
\text { /million }\end{array}$ & $95 \% C I$ & $\begin{array}{l}\text { Incidence/ } \\
\text { million/y }\end{array}$ \\
\hline Amsterdam, Holland ${ }^{16}$ & 1965 & 852500 & 46 & 54 & $40-72$ & 3.1 \\
\hline West Virginia, North America ${ }^{1}$ & 1984 & 555851 & 79 & 142 & $114-177$ & 9.1 \\
\hline Cambridge, UK & 1997 & 684000 & 100 & 146 & $120-178$ & 11.1 \\
\hline Norway $^{11}$ & 1981 & 4107063 & 369 & 90 & 81-99 & 3.8 \\
\hline Denmark $^{12}$ & 1988 & 2300000 & 177 & 77 & $66-89$ & 4.4 \\
\hline Trento, Italy ${ }^{17}$ & 1990 & 444879 & 37 & 83 & $60-115$ & 7.4 \\
\hline Hong Kong ${ }^{18}$ & 1987 & 4860000 & 260 & 53 & $47-60$ & 4.0 \\
\hline
\end{tabular}

cultural, or racial boundaries. However, epidemiological surveys show considerable variation from 1.2/100 000 in Japan in $1958^{4}$ to $17.5 / 100$ 000 in Cyprus in $1994^{5}$; even contemporary studies performed over the past 10 years in developed countries show a threefold difference in prevalence which is unlikely to be explained solely by study methodology and suggests a trend towards rising prevalence over time (table 6). ${ }^{1}$

Here we demonstrate one of the highest prevalence and incidence figures for myasthenia, although our results are in line with recent large population studies from developed countries elsewhere in the world. Confirmation that our estimate of 15/100 000 population represents an increase in disease frequency compared with previous studies from the United Kingdom is made difficult because detailed earlier epidemiological information is lacking. The probable increase is likely to result from increased survival, an increasingly aged population who are at relatively higher risk of developing the disease, a genuine rise in incidence, the application of improved diagnostic tests, and increased recognition of milder cases in which ocular symptoms predominate-features which are all known to inflate prevalence in other neurological illnesses. ${ }^{6}$

Previous reports of myasthenia in the United Kingdom are almost entirely based on the personal experience of the individual neurologist with a particular interest in the disease, or on patients with a selected feature such as thymectomy, each of which is unlikely to be representative. The first epidemiological report from the United Kingdom was from Ferguson et $a l^{7}$ in 1955, who reported on 85 patients diagnosed between 1932 and 1954 from a population of 4.5 million. Later an analysis of 60 patients from the Leeds area ${ }^{8}$ diagnosed between 1934 and
1955 determined a prevalence of $26 /$ million and an incidence of five/million/year. A study of disease frequency in the Merseyside conurbation in $1961^{9}$ found similar figures with a prevalence of $23 /$ million and an incidence of 2.2/million/year. Finally Schon et $a l^{10}$ in 1996 identified 22 patients newly diagnosed in Croydon over a 7 year period and, as in this study, found a large proportion $(13 / 60,22 \%)$ with onset over the age of 60, in marked contrast to Garland and Clark ${ }^{8}$ who identified only $3 / 60(5 \%)$ in this age group.

In all these studies the detailed information required to analyse the impact of improved survival and a rise in incidence or prevalence over time is lacking. In addition, modern epidemiological methodology has undoubtedly improved case recognition. For all these reasons, direct comparison with earlier studies is problematic.

Elsewhere, three large studies are notable for their ability to provide longitudinal incidence data. In a series of detailed studies of the epidemiology of myasthenia in Norway from 1950 to $1982^{11}$ a rise in prevalence was noted from 2.1 to $9.0 / 100000$ but a relatively stable incidence ranging from 2.1 to $4.1 /$ million/year suggesting that the frequency of diagnosing new cases had not changed during the period of the survey. A similar pattern was noted in Denmark, ${ }^{12}$ with prevalence rising from around $20 /$ million in 1970 to $77 /$ million in 1988 whereas incidence was stable at $4.4 /$ million/ year. Lastly, a 25 year survey of myasthenia in Sardinia ${ }^{13}$ saw prevalence rise from 7.7 to 45.0 / million between 1961 and 1986 with incidence at $2.5 / \mathrm{million} /$ year over the same period. Our study suggests a different interpretation. The error is usually to produce a minimum figure when incidence data is derived retrospectively from year of diagnosis and over a relatively short period. Clearly the change in incidence in 
the United Kingdom from 5 and 2.2 in the mid-1950s to $11.1 /$ million in this study seems to indicate that incidence is also contributing to the rise in disease frequency. One striking difference in the subclassification of disease seen in most contemporary studies including our own is the high frequency of ocular disease; this accounted for only $12 \%$ in earlier Scandinavian surveys ${ }^{12}$ and $14 \%$ in a recent analysis of 100 consecutive patients from a neuromuscular clinic in the Netherlands. ${ }^{14}$ This difference may account for the higher overall prevalence in Cambridgeshire and in Virginia, North America. ${ }^{15}$

In summary, we have shown a high prevalence of myasthenia gravis in a previously unsurveyed part of the United Kingdom with nearly a third of cases having onset after the age of 60 years. Disease frequency seems to be rising over time and whereas this is likely to result from improved survival and the advent of new diagnostic techniques, it depends mainly on rising incidence in an increasingly aged population.

1 Phillips LH, Torner JC. Epidemiologic evidence for a changing natural history of myasthenia gravis. Neurology 1996;47:1233-8.

2 Cambridge County Council Research Group. Population forcasts 1989-2001. Cambridge: Cambridgeshire County forcasts 1989-200.

3 Osserman KE, Genkins G. Studies in myasthenia gravis: review of a 20 year experience in over 1200 patients. $M t$ review of a 20 year experience
Sinai f Med 1971;38:497-536.
4 Okinaka S, Reese HH, Katsuki S, et al. The prevalence of multiple sclerosis and other neurological diseases in Japan. Acta Neurol Scand 1966;42:68-76.

5 Kyriallis K, Hristova AH, Middleton LT. What is the real epidemiology of myasthenia gravis [abstract]? Neurology 1995;45:A351-2.

6 Robertson NP, Compston DAS. Surveying multiple sclerosis in the United Kingdom. 7 Neurol Neurosurg Psychiatry 1995;58:2-6.

7 Ferguson FR, Hutchinson EC, Liversedge BA. Myasthenia gravis results of clinical management. Lancet 1955;ii: 636-9.

8 Garland H, Clark ANG. Myasthenia gravis: a personal study of 60 cases. BMF 1956;i:1259-62.

9 Pennington GW, Wilson A. Incidence of myasthenia in the Merseyside conurbation. In: Viets HR, ed. Proceedings of the second international symposium. Springfield, IL: 1961:33745.

10 Schon F, Drayson M, Thompson RA. Myasthenia gravis and elderly people. Age Aging 1996;25:56-8.

11 Storm-Mathisen A. Epidemiology of myasthenia in Norway. Acta Neurol Scand 1984;70:274-84.

12 Somnier FE, Keiding N, Paulson OB. Epidemiology of myasthenia gravis in Denmark. Arch Neurol 1991;48:7339.

13 Giagheddu M, Puggioni G, Sanna G. Epidemiological study of myasthenia gravis in Sardinia, Italy (1958-86). Acta Neurol Scand 1989;79:326-33.

14 Beekman R, Kuks JBM, Oosterhuis HJGH. Myasthenia gravis: diagnosis and follow-up of 100 consecutive patients. f Neurol 1997;244:112-8.

15 Phillips LH, Torner JC, Anderson MS, et al. The epidemiology of myasthenia gravis in central and western Virginia. Neurology 1992;42:1888-93.

16 Oosterhuis HJGH. The natural course of myasthenia gravis: a long term follow up study. $\mathcal{F}$ Neurol Neurosurg Psychiatry 1989;52:1121-7.

17 Ferrari G, Lovaste MG. Epidemiology of myasthenia gravis in the province of Trento (northern Italy). Neuroepidemiology 1992;11:135-42.

18 Yu YL, Hawkins BR, Ip MSM, et al. Myasthenia in Hong Kong Chinese. 1. Epidemiology and adult disease. Acta Neurol Scand 1992;86:113-9. 